\title{
Methanol masers in Galactic center region supernova remnants
}

\author{
Y. M. Pihlström ${ }^{1}$, B. C. McEwen ${ }^{1}$ and L. O. Sjouwerman ${ }^{2}$ \\ ${ }^{1}$ Dept. of Physics \& Astronomy, University of New Mexico, \\ 1919 Lomas Blvd. NE, Albuquerque, NM 87122 \\ email: ylva@unm.edu \\ ${ }^{2}$ National Radio Astronomy Observatory, P. O. Box O, \\ 1003 Lopezville Road, Socorro, NM 87801, USA
}

\begin{abstract}
Methanol masers can be used to constrain densities and estimate kinematical distances to supernova remnants (SNRs), important parameters in cosmic ray acceleration models. With the goal of testing those models both for SNRs inside and outside the Galactic center (GC) region, we have used the Very Large Array to search for $36 \mathrm{GHz}$ and $44 \mathrm{GHz}$ methanol lines in Galactic SNRs. We report on the overall results of the maser search, and in particular the results of the GC SNR G1.4-0.1 in which more than 40 masers were found. They may be due to interactions between the SNR and at least two separate molecular clouds. Methanol masers were also detected in W28 and in Sgr A East.
\end{abstract}

Keywords. masers - (ISM:) supernova remnants — ISM: individual (G1.4-0.1)

\section{Project description and overall results}

To investigate whether methanol masers can be used to constrain the density in the interaction site between SNRs and molecular clouds, 21 SNRs were observed in the 36 $\mathrm{GHz}$ and $44 \mathrm{GHz}$ lines of methanol using the VLA. Both the $36 \mathrm{GHz}$ and $44 \mathrm{GHz}$ transitions were observed, since they trace different density regimes. The VLA highfrequency field-of-view is small compared to the typical SNR sizes, which presents a challenge for the survey. The allocated time only allowed a few pointing positions per target, hence on average $2 \%$ of each SNR was searched for masers. The pointing positions were selected using the positions of previously known $1720 \mathrm{MHz} \mathrm{OH}$ masers, which are known tracers of SNR/molecular cloud interactions.

Despite the limited spatial coverage, over 100 detections were made in Sgr A East, six in W28, and 41 in G1.4-0.1. All detected emission are unresolved point sources, and with the exception of a single pointing position in G1.4-0.1, the lines are narrow consisting of a single spectral feature. The relatively large synthesized beam yielded lower limits of the brightness temperatures of 300-500 K, certainly consistent with thermal emission. On the other hand, many spectral lines are narrower than $0.8 \mathrm{~km} \mathrm{~s}^{-1}$. Combined with the fact that the methanol detections are compact and spot-like, opposed to more widespread thermal emission, it is likely that most detections are maser emission.

\section{The SNR G1.4-0.1}

The SNR G1.4-0.1 contains a large number of methanol masers. Figure 1 shows the angular distribution of the masers overlaid on a $1.4 \mathrm{GHz}$ gray scale continuum map. Less than $8 \%$ of the SNR extent was covered in the observations, implying more masers may be associated with this object. The mean velocities of the masers at each pointing position 


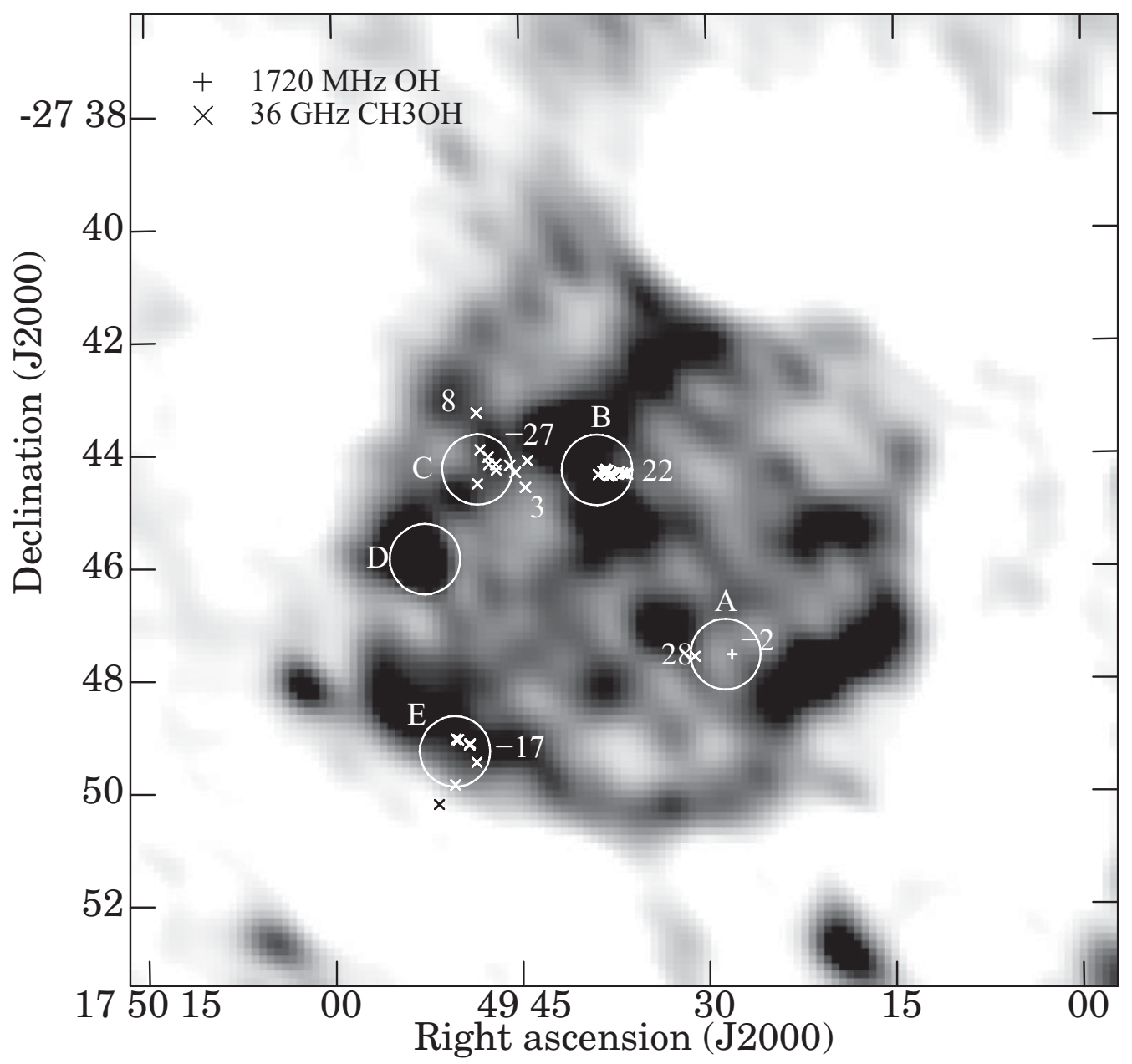

Figure 1. The $36 \mathrm{GHz}$ methanol masers detected in G1.4-0.1 are plotted with crosses, and the single $1720 \mathrm{MHz} \mathrm{OH}$ maser is plotted with a plus symbol. The grey scale is $1.4 \mathrm{GHz}$ continuum map of the SNR. Numbers indicate the mean velocities of the masers in their respective regions. Big circles show the primary beam field-of-view for the methanol maser observations.

differs significantly from the velocity of the single $1720 \mathrm{MHz} O H$ maser at $+2 \mathrm{~km} \mathrm{~s}^{-1}$. The methanol is found over the full $60 \mathrm{~km} \mathrm{~s}^{-1}$ velocity range observed, from about -30 $\mathrm{kms}^{-1}$ to $+30 \mathrm{~km} \mathrm{~s}^{-1}$. Many masers were detected close to either edge of that range, and it is possible that additional masers at higher negative and positive velocities exist.

Two molecular clouds observed in CS (Tsuboi et al. 1999), are found along the lineof-sight toward G1.4-0.1. The velocities of these clouds $\left(\sim-40 \mathrm{~km} \mathrm{~s}^{-1}\right.$ and $\left.40 \mathrm{~km} \mathrm{~s}^{-1}\right)$ agree well with the negative velocity methanol masers observed at the Eastern side of the SNR, and the positive velocity methanol masers at the Western side. There is no unambiguous proof that these molecular clouds do interact with the SNR, although there appears to be a molecular cavity encompassing the SNR especially well outlined in the CS data presented by Tsuboi et al. (1999), similar to the one observed in CO lines in the SNR 3C397 (Jian et al. 2010). Further, given that shocks can produce both an enhanced abundance of methanol and provide a maser pumping mechanism, it seems plausible that the SNR is interacting with both clouds, as previously indicated by the $\mathrm{OH}$ maser. The 
velocity difference between the maser species may be explained by the $\mathrm{OH}$ arising in the post-shock gas, rather than in the shock front region where methanol is found. A similar velocity offset is observed in Sgr A East (Sjouwerman et al. 2010, Pihlström et al. 2011).

\section{Acknowledgements}

The National Radio Astronomy Observatory is a facility of the National Science Foundation operated under cooperative agreement by Associated Universities, Inc. We thank NASA for support under FERMI grant NNX10A055G.

\section{References}

Jian, B., Chen, Y., Wang, J., Su, Y., Zhou, X., Safi-Harb, S., \& Delaney, T., 2010, ApJ 712, 1147

Pihlström, Y. M., Sjouwerman, L. O., \& Fish, V. L. 2011, ApJL 739, L21

Sjouwerman, L. O., Pihlström, Y. M., \& Fish, V. L. 2010, ApJL 710, L111

Tsuboi, M., Handa, T., \& Ukita, N. 1999, ApJSS 120, 1 